Bruche oder einer übertriebenen Falte sich herausbildenden Störungen zu einer steilauffahrenden Bewegungsfläche; wirkt der gebirgsbildende Schub in derselben Richtung konsequent weiter, dann wird die steile Störungsbahn flach, sie wird zur flachen Überschiebung, weil die südlich nachrückende Masse über das vorliegende Gebirge hinüber tritt. Es ist aber ein schlechter Schluß, wenn man aus dem Vorhandensein einer flachen Überschiebung an einer Stelle die Notwendigkeit derselben Störungsform für alle Punkte ableitet. Überschiebungen können eben Aufhängepunkte haben, d. h. Punkte, wo das Höhere mit dem an anderen Stellen tektonisch Tieferen normal, d. h. ohne Unterbrechung durch eine Störung verbunden ist. Für die tirolische Masse sind solche Aufhängepunkte das Gebirge östlich von Kufstein (nördlich vom Kaisergebirge) und das Untertauchen des Wettersteinkalkes östlich vom Sengsengebirge. Dazwischen ist die tirolische Masse auf das nördlich davon Liegende überschoben, und zwar um so mehr, je weiter nördlich die Masse reicht; daher streicht nördlich vom Rauschberg und Kienberg das Bajuvavische unter das Tirolische hinein, während an den Aufhängepunkten der tirolischen Masse Tirolisch und Bajuvavisch nebeneinander liegt.

Diese Erklärung, welche für die ganzen Kalkalpen Anwendung finden kann, macht die Annahme durchstreichender Decken unnotwendig, und sie scheint mechanisch einfacher zu sein. Auf die Unmöglichkeit eines Fernschubes der Kalkalpen wird in der Weiterführung der Erörterung eingegangen werden.

\title{
Der geologische Aufbau des nordwestlichen Afrika.
}

\section{Von E. Jaworski (Bonn.)}

\author{
(Mit 1 Textfigur.)
}

Von zahlreichen kleineren Arbeiten abgesehen, sind in den letzten beiden Jahren zwei umfangreichere Veröffentlichungen französischer Forscher erschienen, die eine sorgfältige Zusammenstellung unserer Kenntnisse über den geologischen Aufbau von Nordwestafrika bringen und sich in glücklichster Weise ergänzen: L. Gentr, »Le Maroc physic «I) und L. Lemoine, 》Afrique occidentale «2). Gentil behandelt die Atlasłänder, und Lemoine Nordwestafrika mit Ausschluß der Atlasländer. Vergleicht man das in großen Zügen wenigstens ziemlich sichere Bild, das diese beiden Forscher entwerfen, mit den spärlichen Angaben, auf welche sich noch SuEss im dritten Bande seines Monumentalwerkes beschränken mußte,

1) F. Alkan, Paris 1912.

2) Handb. d. regionalen Geol. herausg. v. G. Steinmans und O. Wilckens. Bd. 7, Abtl. 6a. Heidelberg 1913. 
so fällt der gewaltige Fortschritt in der geologischen Erforschung dieses Gebietes in den letzten 10 Jahren auf. Dieser Umstand mag die kurze Zusammenstellung, wie sie im folgenden versucht ist, rechtfertigen. Sie beschränkt sich auf das Gebiet nördlich des $15^{\circ} \mathrm{n}$. Br., der ungefähr mit der Senegalmündung und der Südgrenze des Zentralplateaus von Touareg zusammenfällt und westlich des $12^{\circ}$ w. L., der etwa mit der Ostküste von Tunis abschneidet. . Weiter im Süden sind unsere Kenntnisse noch zu spärlich, um eine auch nur ungefähre Vorstellung vom genaueren Aufbau des Landes geben zu können.

Schon äußerlich zerfällt das Gebiet von N. nach $\mathrm{S}$. in drei Teile: 1. die Gebirgsländer des Atlas (bis $4500 \mathrm{~m}$ ), 2. das cretacische Tafelland der Sahara, 3. das zum großen Teil aus flach liegendem Paläozoicum bestehende Zentralplateau von Touareg (bis $2200 \mathrm{~m}$ ) im O. und Mauretanien im W., mit Ausnahme des Küstencaumes. Eine scharfe Grenze gegen das im S. folgende Senkungsgebiet des Sudan scheint vor allem im W. zu fehlen. Wie sich ergeben wird, unterscheiden sich diese drei Teile auch durch ihren inneren Aufbau.

Granite und kristalline, z. T. meta morphe Gesteine archäischen Alters sind im ganzen Gebiet bekannt: im Zentralplateau von Touareg, wo sie eine ausgedehnte Peneplainlandschaft bilden, im Hinterland von Mauretanien und in den Atlasländern. Eine ähnlich weite Verbreitung hat das stellenweise (Sahara) fossilführende Silur, das aus Schiefern und Quarziten besteht und in metamorphem Zustande oft nur schwer von archäischen kristallinen Schiefern zu trennen ist. Silurisch sind wohl auch alte Schiefer im Hinterland von Rio del Oro und metamorphe Glimmerschiefer, Quarzite und Sericitschiefer im südlichen Mauretanien (Adrar Tmar und Tagant). Gegen Ende des Silur erfolgte eine starke Gebirgsbildung, die im Zentralplateau und in Mauretanien deutlich zu beobachten ist. Sie läßt sich weit nach S. verfolgen und hat im $\mathrm{N}$. wahrscheinlich auch noch das Atlasgebiet mit ergriffen, scheint also das ganze Gebiet betroffen zu haben. Diese Faltung führte zur Entstehung NS.-streichender Ketten, der SuEssschen Sa hariden (älter als Obersilur), die den Kaledoniden Nordeuropas entsprechen und auch ungefähr mit der takonischen Faltung des östl. Nordamerika gleichalterig sind Das gleichfalls im ganzen Gebiete bekannte De von legt sich über die denudierten und erodierten Sahariden und ist in der Sahara stellenweise sehr fossilführend und vorzüglich gegliedert. Zwischen Adrar Tmar und Zentralmassiv Touareg fehlt es, dort liegt Carbon auf Silur. Eine zweite große Faltung führt postdevonisch zur Entstehung mächtiger Gebirge, der "Altaides Africaines «, die den armorikanisch-variscischen Gebirgen Europas entsprechen. Diese Faltung hat den S. des Gebietes nicht mehr ergriffen, im Zentralplateau Touareg liegen Devon und Carbon horizontal und ebenso im südl. Mauretanien, während im nördl. Miauretanien anscheinend (Lemorne, S. 24, Fig. 5) das Carbon noch mitgefaltet ist. In diesem Verhalten liegt der grundlegende Unterschied des 
südlichsten Gebietes gegenüber den weiter nördl, gelegenen Teilen: das erstere ist im Gegensatz zu letzteren postsilurisch nicht mehr gefaltet worden. Während im $O$. die Grenze des postsilurisch nicht mehr gefalteten Plateaus Touareg gegen die Sahara sehr scharf ist, läßt sich im W. zurzeit diese Grenzlinie noch nicht mit derselben Genauigkeit angeben. Sie liegt aber, wie das erwähnte gefaltete Carbon zeigt, jedenfalls südlicher als die auf der Karte eingetragene Nordgrenze der Verbreitung des Paläozoicums, die aus diesem Grunde in ihrem westl. Teil gestrichelt ist, und zieht vielleicht in der Gegend des Adrar Tmar durch. Das Alter dieser zweiten Faltung ist im Atlas jünger als Dinantien und älter als oberes Perm, genau wie in Mitteleuropa. In der Sahara im Gebiet von Saoura liegt Carbon -- leider ist kein genaues Alter angegeben, der Karte nach (Lemoine, S. 24) anscheinend Dinantien - horizontal auf Devon. Dann würde die Faltung im S. etwas älter sein als im N., also ein Wandern der Faltung, wie es ja auch in Europa bekannt ist, vorliegen. Diese Altaiden haben in der südl. Sahara ein submeridionales Streichen, worin man vielleicht eine Hindeutung auf ihre posthume Natur in bezug auf die Kaledoniden sehen kann. Ein interessantes Scharungsgebiet ist im Atlas das Plateau von Siroua: westlich davon herrscht variscisches, östl. armorikanisches, und bei Siroua selbst meridionales Streichen. Der Bau ist ein vollkommenes Analogon zu der Scharungsregion des französischen Zentralplateaus. Die Altaiden verliefen also etwa parallel, nicht senkrecht zur jetzigen Küste wie das heutige Gebirge. Das Scharungsgebiet zeigt starken Metamorphismus der Gesteine und ist reich an prämesozoischen granitischen Intrusionsn. Eine interessante Bifurkation des variscisch-gerichteten NNO.-streichenden Armes ist aus der marokkanischen Meseta im Lande von Chaouia bekannt: der variscische Ast teilt sich in einen nach NNW. und einen nach ONO. gerichteten $Z$ weig, von denen sich der erstere bis zum atlantischen Ozean, der letztere bis zum mittleren Atlas verfolgen läBt. Es folgt eine rasche Einebnung des Gebirges zu einer Peneplain, deren Reste in der Sahara, in der marokkanischen und algerischen Meseta bekannt sind. Auf ihr wurden rote Sandsteine und Konglomerate terrestrer Entstehung von permo-triadischem Alter abgelagert. Marines Perm, Trias, Jura und untere Kreide fehlen in der Sahara südlich der Atlasländer, so daß für dieses Gebiet vom Perm bis zur mittleren Kreide eine Festlandperiode anzunehmen ist. Es iš̀ das ein grundlegender Unterschied gegenüber den Atlasländern, wo marine Trias, vor allem aber Jura und Kreide reich entwickelt sind. Wie die Sahara verhält sich die marokkanische Meseta, nur daß dort marines Rät bekannt ist, während in der algerischen Meseta und in dem Tafelland nördlich des östlichen mittleren Atlas reichlich flachliegender Jura vorhanden ist. Im Atlasgebiet wurde die Peneplain bald durch Brüche zertrümmert, zwischen denen ausgedehnte Senkungsregionen, deren Lage ungefähr mit dem heutigen Hohen Atlas, Mițleren Atlas und dem Saharaatlas zusammenfällt, in die Tiefe sanken. Die Bruchbildung 
war von dem Empordringen gewaltiger Massen permotriadischer Eruptivgesteine begleitet (Basalte, Trachyte). Während das mesozoische Meer im Westen auf die Bruchzone zwischen marokkanischer Meseta und Sahara beschränkt war, griff es im Osten aus der Geosynklinale des Saharaatlas über den Bruchrand nach Norden auf die algerische Meseta über.

Verfolgen wir zunächst die weitere Geschichte der Sahara. Kurz vor der mittleren Kreide transgredierte das Meer von neuem und behauptete sich mindestens noch während des ganzen Eocäns. Wie weit das Meer nach Süden reichte, ist schwer zu sagen. Die Kreideschichten können von großen Teilen des Gebietes, wo jetzt Paläozoicum zutage tritt (Plateau Touareg, Mauretanien) durch Erosion entfernt sein. Wahrscheinlich ist aber doch wenigstens das Plateau Touareg zum Teil nicht mehr vom Kreidemeer überflutet worden. Die große tertiäre Gebirgs bildung greift nach Süden nicht über die A tlasländer hinaus, sie fehlt in der Sahara, von ganz unbedeutenden Ausklängen a bgesehen. Hierin liegt ein zweiter wichtiger Unterschied zwischen der Sahara und den Atlasländern. Die heutige Grenze der Sahara gegen das Plateau Touareg im Süden und gegen den marokkanischen Hohen Atlas im Norden werden von jungen postcretacischen Verwerfungen gebildet, die mit der Südgrenze der herzynischen bez. tertiären Faltung zusammenfallen.

Von der mittleren Kreide ab ist das ganze Gebiet der Atlasländer auch die marokkanische Meseta - wieder vom Meere bedeckt. Postjurassisch erfolgt die erste Heraushebung eines Teiles der Atlasgeosynklinale in der Gegend des Zentralmassives des Hohen Atlas, die erste Anlage des heutigen Atlas. Im Westen im Küstengebiet des Hohen Atlas liegt auf Obereocän direkt Tortonien und Plaisancien: In die Zeit der Lücke fällt die tertiäre Gebirgsbildung, die allerdings im äußersten Westen auch noch die letztgenannten Stufen ergriffen hat. Diese tertiäre Auffaltung, die zur Entstehung des heutigen Gebirges geführt hat, erfolgte im Bereich des durch die postherzynischen Brüche bereits vorgezeichneten Senkungsgebietes, dehnt sich aber nicht auf die marokkanische und algerische Meseta im Norden der Senkungsgebiete aus. Diese haben sich vielmehr wie die Sahara verhalten und sind nur von Vertikalverschiebungen betroffen worden. Zur gleichen Zeit wird im Norden in dem zur Mittelmeerregion gehörigen Geosynklinalgebiet der Tellatlas aufgefaltet. Auf die Faltung folgt Bruchbildung, welche eine Wiederbelebung der alten postherzynischen Spalten bedeutet, welche die Atlasgeosynklinale begrenzten. Diese Brüche begrenzen im Norden und Süden das System des Hohen Atlas mit dem Mittleren Atlas und Antiatlas gegen die abgesunkene marokkanische Meseta und die Sahara. Hiermit verbunden ist das Empordringen junger Eruptivgesteine und die Bildung neogener Vulkane in dem alten herzynischen Zerrüttungs- und Scharungsgebiet von Siroua (Trachyt, Phonolith, Andesit). Auch im Tellatlas ist im Anschluß an die Gebirgsbildung vulkanische Tätigkeit bekannt in der 
Gegend von Oran und bei Angad mehr im Süden. Die jungen Brüche haben auch das Plateauland der marokkanischen und algerischen Meseta wie auch der Sahara zerstückelt.

Zum besseren Verständnis der tertiären Gebirgsbildung sei eine kurze orographische Übersicht vorausgeschickt. Von dem südlichen Teil der tunesischen Ostkiuste zieht nach WSW. der Saharaatlas, in dessen Verlängerung der Hohe Atlas liegt, der zwischen Kap R'Ir und Agadir den atlantischen Ozean erreicht und in seiner Streichrichtung auf die Kanarischen Inseln stößt. Vom Hohen Atlas zweigt nach SW. der Antiatlas $a b$, der gleichfalls in den Ozean ausstreicht. Zwischen beiden liegt das Tal von Sous. Nach NO. zweigt vom Hohen Atlas der Mittlere Atlas ab. Im nördlichen Algier zieht an der Mittelmeerküste entlang von Tunis aus, wo er mit dem Sahara-Atlas zusammenstößt, bis in die Gegend von Melilla der Tellatlas. Zwischen Tellatlas und Saharaatlas liegt die algerische Meseta, deren westl. Zipfel sich in den Winkel zwischen Mittleren Atlas und östl. Hohen Atlas einzwängt. Im S. stoßen Saharaatlas und Hoher Atlas an das Saharaplateau, das im W. als Plateau von Draa und Tafilelt bezeichnet wird. In der Plateauregion finden sich nur vereinzelte, leicht aufgewölbte Antiklinalzüge, deren bedeutendster der Djebel Bani ist. Im N. des westl. Hohen Atlas und im NW. des Mittleren Atlas liegt die marokkanische Meseta. SchlieBlich verläuft im N. parallel der Küste zwischen Melilla und Ceuta der Gebirgszug des Riff. Zwischen dem Riff einerseits und der marokkanischen Meseta im W. und dem Nordabfall des Mittleren Atlas andererseits liegt die mit jungtertiären Sedimenten aufgefüllte Riffenge.

Der Hohe Atlas mit seinen Nebenketten, dem Antiatlas und dem Mittleren Atlas ist gegen N. wie gegen S. durch Brüche gegen das Plateauland abgegrenzt. Das Tal von Sous zwischen dem Hohen Atlas und dem Antiatlas ist ein nach Norden wie nach Süden gestaffelter Grabenbruch. Der Hohe Atlas besteht aus drei parallelen Antiklinalketten (in der Küstengegend nur noch zwei), deren Achse sich nach der Küste zu sehr rasch senkt und unter den atlantischen Ozean untertaucht. In seiner Längserstreckung gliedert er sich in ein östliches und westliches Antiklinalmassiv, die durch eine synklinale Senkungsregion getrennt sind. Die Hauptphase der bereits zur Kreide einsetzenden Gebirgsbildung (Zentralmassiv des Hohen Atlas) fällt in das Miocän, dauert aber namentlich in der Küstenregion noch fort bis ins Plaisancien. Von Westen nach Osten nimmt die Höhe des Gebirges ab, und es treten immer jüngere Schichten zutage. Die gebirgsbildende Kraft hat von Norden nach Süden gegen die Sahara als Vorland gewirkt, wie die stellenweise überkippten oder auch überschobenen Antiklinalen des Küstengebietes, sowie im Westen und Osten des Hohen Atlas zeigen. Die in der Streichrichtung des Hohen Atlas liegenden Kanaren bestehen oberflächlich größtenteils aus jungvulkanischen Gesteinen. Die aber von ihnen bekannten Vorkommen alter Gesteine wie Gabbro, Diorit, Syenit metamorphe Tonschiefer und 
fossilführendes Cenoman beweisen, daß die Reste eines Gebirgsmassives älterer Entstehung vorliegen, das fast ganz durch junge Bildungen verhüllt ist. Gevrru sieht in den Kanaren ein versunkenes Massiv analog dem östlichen oder westlichen Massiv des Hohen Atlas, das durch eine Senkungsregion, wie sie auch zwischen den Massiven des Hohen Atlas liegt, vom Festland getrennt ist.

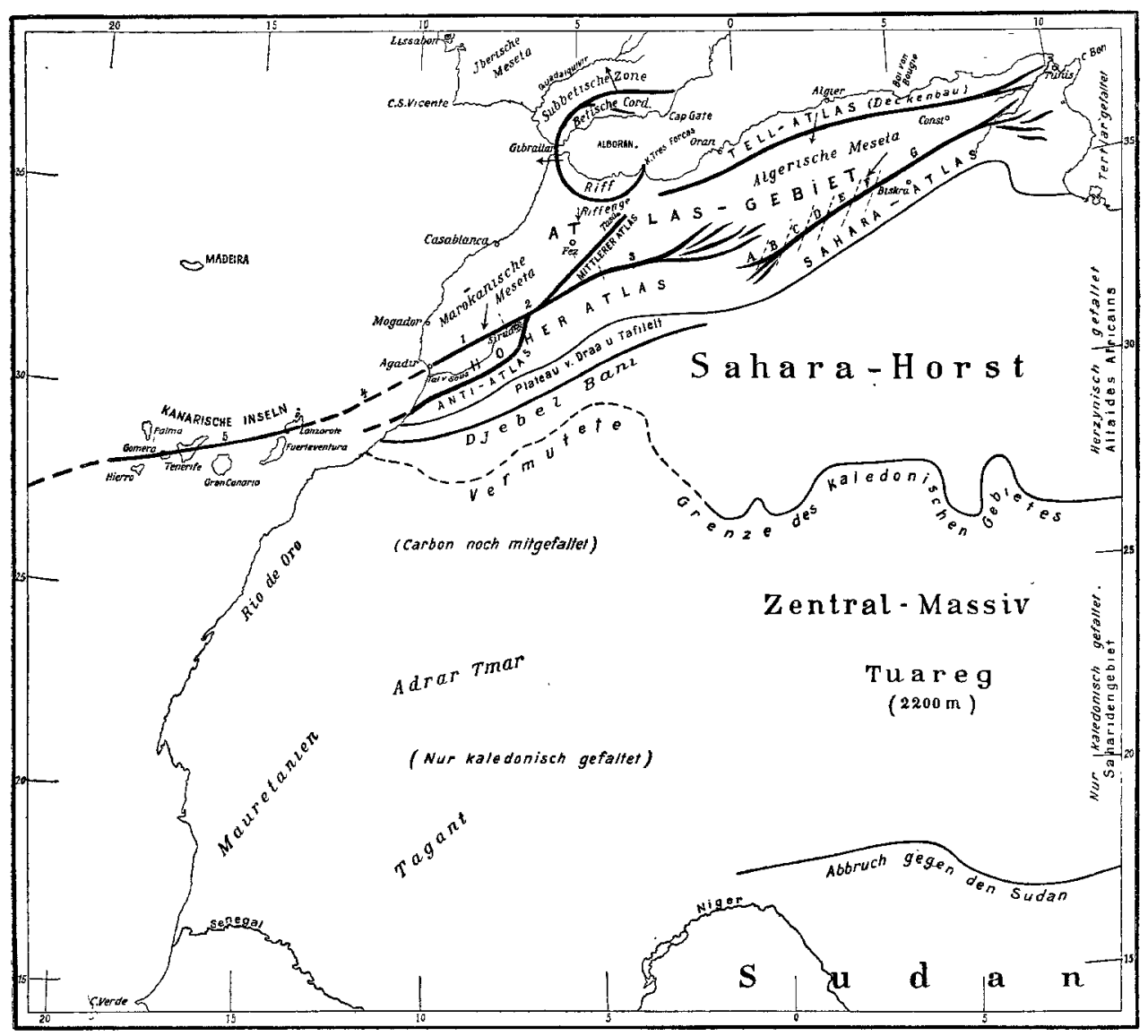

Fig. 1. Schematische geologisch-tektonische Karte des nordwestlichen Afrika nach den Arbeiten von Lemorne und GentiL. Maßstab etwa $1: 26000000$.

Es bedeutet im Bereiche des Hohen Atlas: 1 = westliches Antiklinalmassiv, $2=$ mittlere synklinale . Senkungsregion, $3=$ östliches Antiklinalmassiv, $4=$ Syn . klinalregion zwischen dem afrikanischen Festland und dem Kanarenmassiv, $\mathbf{5}=$ Antiklinalmassir der Kanaren. Es bedeutet im Gebiet des Saharaatlas: $A=$ Synklinalregion zwischen den östlichen Ausläufern des Hohen Atlas und dem Massiv des Ksour, $B=$ Antiklinalregion des Massiv des Ksour, $C=$ synklinale Senkungsregion zwischen dem Massiv des Ksour und dem Massiv d'Amour, $D=$ Antiklinalmassiv, d'Amour, $E=$ synklinale Senkungsregion zwischen dem Yassiv d'Amour und dem Massiv Oulad Nayl, $F=$ Antiklinalmassiv des Oulad Nayl, $G=$ Djebel Aurès.

Die Pfeile geben die Schubrichtung an. 
Der durch die Querkette des Djebel Siroua mit dem Hohen Atlas zusammenhängende Antiatlas besteht gleichfalls aus Ketten von antiklinalem Bau, deren Achse sich nach Westen unter das Meer senkt. In der Gegend der Synklinalregion zwischen den beiden Massiven des Hohen Atlas zweigt nach NO. der Mittlere Atlas ab, aus drei Antiklinalketten bestehend, die mit starker Senkung der Achse unter die jungtertiären Absätze der Riffenge untertauchen. Der Saharaatlas besteht nicht aus zusammenhängenden Antiklinalkämmen, sondern aus drei von Westen nach Osten folgenden gegen $\mathrm{N}$. staffelförmig gegeneinander verschobenen 'kulissenartigen Massiven: Massiv Ksour, Massiv d'Amour, Massiv Oulad Nayl. Die Massive sind durch Senkungsregionen getrennt. Sie bestehen aus einzelnen Antiklinalen, welche etwas mehr meridional verlaufen als die Streichrichtung des Gebirges und sich bündelartig zusammenschließen. Die Faltenbündel sind von SW. nach NO. in der Weise staffelförmig angeordnet, daß sie mit ihrem SW.-Teil noch in der Südhälfte eines Massivs liegen, während sie mit ihrem NO.-Teil schon an der Zusammensetzung der Nordhälfte des nächsten Massives teilnehmen. Die einzelnen Antiklinalen sind nach Süden gegen die Sahara als Vorland überkippt oder überschoben wie im Hohen Atlas. Noch weiter nach Osten finden sich girlandenartige Antiklinalketten gegen das Kap Bon hin. Der Hohe Atlas breitet sich an seinem Ostende in vielen Zügen fächerförmig in der Ebene aus, mit starker Senkung der Achse. Weiter im Osten steigen in der gleichen Weise die Antiklinalzüge des Massiv Kour aus der Ebene auf, und es besteht kein Zweifel, daß das Trennungsgebiet zwischen Hohem Atlas und Saharaatlas nur einer Senkungszone entspricht, wie sie auch die einzelnen Massive des Saharaatlas trennt, daß also der Hohe Atlas und der Saharaatlas untereinander zusammenhängen und sich gegenseitig nicht ferner stehen wie die einzelnen Massive des letzteren untereinander. Hierfür spricht auch der Umstand, daß antiklinale Ketten, die im Süden des Hohen Atlas aufsetzen, in ihrer Verlängerung bereits auf der Nordseite des Massiv Ksour liegen, genau wie die Kulissenfalten im Saharaatlas. Betrachtet man die ganze zusammenhängende Kette des Hohen Atlas und des Saharaatlas von den Kanaren bis zur tripolitanischen Küste, so erscheint es sehr wahrscheinlich, daß auch das östliche und westliche Massiv des Hohen Atlas genau wie im Saharaatlas aus kulissenartig angeordneten Faltenbündeln bestehen, und daß auch die Senkungsregion zwischen dem Festland und dem Massiv der Kanaren den gleichen Bau besitzt wie die Senkungsgebiete zwischen den einzelnen Massiven des Saharaatlas Hierfür fehlen aber noch die nötigen Beobachtungen. Der ganze ausgedehnte Gebirgszug nimmt gesetzmäßig von Westen nach Osten an Höhe $a b$, die Achse senkt sich immer mehr, und es treten immer jüngere Gesteine zutage: im Hohen Atlas Paläozoicum, kristalline Gesteine und Jura, im Massiv Ksour Triaskerne mit Jura und Kreide und ganz im Osten nur noch Kreide und Tertiär mit ganz wenig Jura. 
Der Tellatlas an der Mittelmeerküste hängt im Osten mit dem Saharaatlas zusammen, während sich im Westen seine Achse unter das Tertiär der Riffenge senkt und sich im Mittleren Atlas fortsetzt, der auf der anderen Seite der Riffenge aus dem Tertiär auftaucht. Während im Tellatlas das Mesozoicum bathyale Facies besitzt, ist es in den beiden anderen Teilen neritisch ausgebildet. Außerdem steigert sich im Tellatlas die Gebirgsbildung bis zur Bildung ausgedehnter liegender Falten (bis $15 \mathrm{~km}$ ) und zum Deckenbau, während sie in den übrigen Teilen des Atlasgebirges sich auf normale Faltung beschränkt. Die Deckenbildung erfolgte zwischen Mittel- und Obermiocän, da ersteres von Lias überschoben ist, und letzteres bereits über die Decken transgrediert. Die Schubrichtung ist gegen Süden gerichtet, gegen die algerische Meseta als Vorland und klingt gegen Süden langeam in Überschiebungen, Falten und Brüche aus. Alte kristalline Massive, den Zentralmassiven der Alpen vergleichbar, sind an der Nordküste bekannt, z. B. an der Bai von Bougie. Die Wurzeln der Decken liegen im Mittelmeer.

Es ist wiederholt davon gesprochen worden, daß die Horste der algerischen und marokkanischen Meseta nicht an der tertiären Auffaltung teilgenommen haben, sondern nur durch Brüche zerstückelt sind. Wenn dies auch im allgemeinen zutrifft, so sind andererseits doch auch in den Tafelländern schwache Faltungen zu erkennen, welche als Ausklänge der gebirgsbildenden Kraft, die die zwischenliegenden Synklinalgebiete gefaltet hat, auf die Horste zu erklären sind, "repercussion des mouvements «. So ist im N. in der marokkanischen Meseta eine Reihe kurzer Antiklinalen und Synklinalen bekannt und ebenso im. Süden in der Sahara. Ein solcher antiklinaler Hügelzug ist der Djebel Bani, der sich vom atlantischen Ozean auf eine Strecke von $700 \mathrm{~km}$ im S. des Hohen Atlas verfolgen läßt. Ebenso greift im Tellatlas die Gebirgsbildung auf die algerische Meseta über und klingt erst allmählich nach Süden aus. Es folgen von Norden nach Süden: Decken, Überschiebung, Faitung, Verwerfung. Neben der im allgemeinen nach Süden gerichteten Faltung finden sich auch lokal Falten, die von Süden nach Norden gelegt sind, und die als Rückfaltung im Sinne von Suess aufzufassen sind. Solche Rückfaltungen finden sich am Südrand der marokkanischen Meseta und sind dadurch zustande gekommen, daß die im Norden gelegene marokkanische Meseta gegenüber dem im Süden gelegenen Saharahorst in die Tiefe gesunken ist. Auch in der Sahara selbst findet sich eine Reihe ganz schwacher Synklinalen und Antiklinalen, die splis posthumes d'âge atlasique von Lemorve, die in zwei verschiedenen Hauptrichtungen streichen und posthume Bildungen auf präexistierenden herzynischen Faltensystemen repräsentieren sollen.

Wir sehen also, wie der Hohe Atlas nebst seinen Nebenketten (Antiatlas und Mittlerer Atlas) mit dem Tellatlas und dem Saharaatlas zusammenhängt. Wir wissen, daß ihre Hauptbildung gleichzeitig erfolgt ist, wenn sie vielleicht auch in dem einen Teil frïher begonnen oder länger 
angedauert hat, wie in dem anderen. Wir wissen ferner, daß die Faltung überall gleichsinnig von Norden nach Süden gegen das Saharaplateau als Vorland gerichtet ist, wenn sie auch graduell verschieden stark gewesen ist, so daß die Kraft in dem einen Teil zur Ausbildung yon Überschiebungsdecken geführt hat, während sie in den anderen Teilen nur zur Entstehung von Antiklinalen und Synklinalen geführt hat. Bii dieser großen Übereinstimmung des Bauplanes liegt zweifellos auch eine einheitliche Entstehungsursache für das ganze Atlasgebiet zugrunde, die sich LEMorNe folgendermaßen vorstellt: eine von Norden wirkende Kraft hat die widerstandsfähige marokkanische und algerische Meseta gegen den Saharahorst gepreßt und hierbei die schon seit dem Permocarbon schwachen Synklinalzonen des Hohen Atlas und Saharaatlas aufgefaltet. Die marokkanische Meseta ist entsprechend dem ungefähren OW.-Streichen des Hohen Atlas annähernd in nordsüdlicher Richtung bewegt worden, während man bei der algerischen Meseta entsprechend dem NO.-SW.-Streichen und den kulissenartig angeordneten Faltenbündeln des Saharaatlas, nicht reine NS.-Bewegung, sondern auch eine Seitenverschiebung von NO. nach $\mathrm{SW}$. annehmen muß. Durch die verschiedene Bewegungsrichtung der algerischen und marokkanischen Meseta wurde auch die zwischen den beiden liegende schwache Region zusammengepreßt, und so erklärt sich die Entstehung des mittleren Atlas. Nach GentiL ist die marokkanische Meseta etwa um $25 \mathrm{~km}$ nach Süden verschoben worden, und der $\mathrm{Zu}$ sammenschub in der Gegend des Mittleren Atlas entspricht dem gleichen Betrag. Diese Kraft, die vom Mittelmeer gegen den Saharahorst gewirkt hat, ist die gleiche, die die Synklinale des Tellatlas zu einem Gebirge von Deckenbau aufgefaltet hat. Man kann sich denken, daß diese Kraft sich dann auf die algerische Meseta iibertrug, die letztere Masse aber nicht überwältigen konnte, sondern als einen einheitlichen Körper von NO. nach SW. verschob und erst wieder die südlich gelegene Synklinale des Saharaatlas zusammenstauchte. Die Gebirgsbildung ist gleichaltrig mit der Alpenfaltung, und es sei daran erinnert, daß der Tellatlas die Fortsetzung des Apennins ist.

Das genaue Spiegelbild der östlichen Atlasländer (Tellatlas, algerische Meseta, Saharaatlas und Saharahorst) findet sich in Zentraleuropa im Alpenbogen, Schweizer Mittelland, Juragebirge und französischen Zentralplateau. Es entsprechen sich die Glieder in der Folge, wie sie aufgeführt sind. In Afrika hat die Kraft von Norden gegen das im Süden liegende Vorland gewirkt, während in Europa die Kraft von Süden gegen das im Norden liegende Widerlager stößt. Die bathyalen Sedimente der Alpen sind gegen das flachliegende Schweizer Mittelland als Vorland in Decken gelegt, die bathyalen Sedimente des Tellatlas sind gegen das Vorland des algerischen Horstes mit flacher Lagerung der Schichten in Decken gelegt. Die neritischen Sedimente des vom Alpenbogen sich abzweigenden Schweizer Jura, der das Vorland, das Schweizer Mittelland, auf der anderen Seite begrenzt, sind in einfache Antiklinalen auf- 
gefaltet. Die neritischen Sedimente des vom Tellatlas sich abzweigenden Sahara-Atlas, der die algerische Meseta auf der anderen Seite einschließt, sind ebenfalls in einfache Antiklinalen gelegt. Den alten kristallinen Massiven der Alpen entsprechen die kristallinen Massive der algerischen Küste, die allerdings zum größten Teil im Mittelmeer versenkt sind. Das französische Zentralplateau bildet in dem einen, der Saharahorst in anderen Falle das Widerlager, gegen das die gebirgsbildende Kraft geschoben hat.

Das Riff hängt nicht mit dem Atlasgebirge direkt zusammen und setzt sich nach Osten nicht in den Tellatlas fort, sondern streicht in das Mittelmeer aus. Es besteht im Westen aus einem Kamm von Jura. Nach der Mittelmeerküste folgen ältere Gesteine bis ins Kristalline, nach dem Atlas zu jüngere Schichten bis ins Eocän. Es besteht aus rosenkranzartig aneinander gereihten Antiklinalkuppeln und hängt im W. über die Meerenge von Gibraltar unzertrennlich mit den spanischen Gebirgszügen zusammen. Die Meerenge von Gibraltar entspricht nur einer starken Senkung der Riffachse.

Auf der europäischen Seite unterscheidet man zwischen der äußeren, am Mittelmeer gelegenen Betischen Kordillere, die aus paläozoischen und mesozoischen ziemlich stark metamorphen Sedimenten besteht, ohne Deckenbau, und der nach dem Guadalquivirtal zu folgenden subbetischen Zone aus Tertiär und Mesozoicum mit Deckenbau. Es fragt sich, ob das Riff in beide Zonen fortsetzt oder nur in eine derselben und in welche. Mit Sicherheit läBt sich meines Wissens die Frage zurzeit nicht beantworten. GEnTIL spricht nur von einer Fortsetzung in die betische Kordillere, doch scheint die bis in das Eocän reichende Sedimentserie und der auf der Außenseite des Riffs bekannte Schuppenbau dafür zu sprechen, daß im Riff mindestens auch Äquivalente der subbetischen Zone vorhanden sind. In dem ganzen Gebirgsbogen ist die Bewegung nach außen, das heißt nach der konvexen Seite des Bogens gerichtet. Auch die Masse der betischen Kordillere ist nach außen, also gegen das Guadalquivirtal hin bewegt worden. Im Riff ist die Bewegung von Norden nach Süden und nicht umgekehrt gerichtet. GENTIL nimmt auf die Tiefenverhältnisse in diesem Teil des Mittelmeeres und auf die Ähnlichkeit der Eruptiva auf der Insel Alboran und im Ostteil des Riff gestützt an, daß die betische Kordillere auch im Osten über das Kap de Gata, die Insel Alboran und das Kap Tres Forkas mit dem Riff zusammengehangen hat. Er spricht von einem ringförmig geschlossenen Kranz junger Faltengebjrge um eine alte Masse, die zum größten Teil im westlichen Mittelmeer versunken ist. $\mathrm{Zu}$ dieser würde voraussichtlich auch das alte Gebirge der betischen Kordillere gehören. Diese Auffassung ist unwahrscheinlich. Viel wahrscheinlicher ist, daß die Masse der betischen Kordillere nach Osten im Mittelmeer ausstreicht und im Süden der Balearen, der Fortsetzung der subbetischen Zone, im Meere versunken fortstreicht. Eine ganz andere Auffassung vertritt TERMIER und mit ihm KoBER, die in der marokkani- 
schen Meseta eine alte Zwischenmasse zwischen dem Atlas-ApenninStamm und den "Chaînes alpines « sehen und Decken annehmen, die bereits in der Riffenge wurzeln und von dort von Süden nach Norden und nicht umgekehrt geschoben worden sind.

Die Riffenge ist eine jungtertiäre Senkungszone zwischen dem Riff und der marokkanischen Meseta, bzw. dem Nordostabhang des Mittleren Atlas. Sie entspricht einer alten Meeresverbindung, die jünger ist als die Verbindung durch die Nordbetische Enge und älter als die Verbindung über Gibraltar. Man kann verfolgen, wie seit dem Untermiocän vom Mittelmeer wie vom atlantischen Ozean her das Meer nach der Gegend von Taza transgrediert, und wie gegen das Pliocän hin die Verbindung allmählich aufhört.

Die Azoren sind nach GenTIL der westlichste Vorposten des Atlas und bilden das Bindeglied zwischen den mediterranen tertiären Faltenzügen und den Faltenzügen Mittelamerikas. Es sei betont, daß diese Theorie mit der Ansicht von SuEss nicht übereinstimmt. Dieser nimmt an, da $\beta$ der östliche Kordillerenbogen über Trinidad in den Antillenbogen ïbergeht, und sagt nichts von einem ja immerhin möglichen Ast, der nach Osten in den Ozean ausstreichen und die Verbindung mit dem Atlas herstellen könnte. Ich gedenke in einem anderen Aufsatz auf diese Frage näher einzugehen. Wir sehen also, wie sich in Afrika von Süden nach Norden gegen das alte Tethysgebiet zu immer jüngere Faltungsperioden ablösen, bzw. wie die jüngeren Faltungsperioden immer weniger weit nach Süden übergreifen. In Europa finden wir das Spiegelbild dazu: auch hier wandern die jüngeren Faltungen gegen das Tethysgebiet zu, hier allerdings von Norden nach Süden. In Skandinavien-Schottland findet sich die kaledonische Faltung und keine herzynische Faltung mehr, in Mitteleuropa die herzynische Faltung und in den Alpenländern die tertiäre Gebirgsbildung. Es ist jedoch unwahrscheinlich, daß die älteren Faltungen ursprünglich auch nur auf das Gebiet beschränkt gewesen sind, in welchem wir sie jetzt kennen; wir wissen, daß in Afrika die Sahariden weit über das Bereich des Plateaus Touareg nach Norden gegriffen haben. Dasselbe trifft zweifelsohne auch für die Kaledoniden zu, die auch nach Zentraleuropa übergegriffen haben, dort aber, weil durch jüngere, im Norden nicht mehr vorhandene Faltung verwischt, naturgemäß nur sehr schwierig zu erkennen sind. 